\title{
Programação remota dos sistemas de implante coclear
}

\section{Remote programming of the cochlear implant systems}

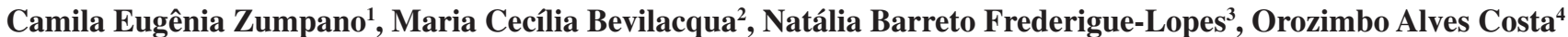

\begin{abstract}
RESUMO
O objetivo deste estudo foi investigar os recursos necessários para a viabilização da programação remota dos sistemas de Implante Coclear (IC) e verificar os benefícios e limitações para a implementação deste modelo de atendimento nos serviços de saúde auditiva do Brasil. Participaram dois usuários de IC multicanal acompanhados no Centro de Pesquisas Audiológicas (CPA) do Hospital de Reabilitação das Anomalias Craniofaciais (HRAC) da Universidade de São Paulo (USP) e dois profissionais que atuam na área de Audiologia. Um dos profissionais estava localizado no CPA/HRAC-USP, denominado Centro de Referência (CR) e outro localizado em um centro situado a mais de $1500 \mathrm{~km}$ de distância do CR, denominada Área Remota (AR), sem experiência prévia na programação deste dispositivo. O procedimento consistiu no treinamento do recurso selecionado para a programação remota entre o CR e a AR. Após a execução da teleconsulta, o profissional da AR e os sujeitos implantados responderam a um questionário previamente elaborado. O estudo revelou que dentre as vantagens da aplicação deste procedimento estão a descentralização dos serviços de saúde auditiva, o treinamento e capacitação de profissionais, a redução de custos para o Sistema Único de Saúde (SUS) e o acompanhamento sistemático de pacientes implantados. A teleconsulta é um modelo de acompanhamento dos sujeitos implantados efetivo, viável e inovador no Brasil. Entretanto, ainda é necessário realizar mais pesquisas para viabilizar o uso em escala nacional.
\end{abstract}

Descritores: Consulta remota/métodos; Implante coclear; Perda auditiva; Telemedicina/instrumentação; Relatos de casos

\section{INTRODUÇÃO}

A Teleprática consiste na aplicação da tecnologia das telecomunicações para fornecer serviços profissionais à distância, ligando o profissional ao cliente ou a outro profissional com a finalidade de realizar avaliação, intervenção e/ou consulta ${ }^{(1-3)}$. Outros termos para se referir a essa forma de prestação de serviços são: Telemedicina ${ }^{(4-5)}$ e Telessaúde ${ }^{(5)}$. O primeiro, destinado exclusivamente à aplicação na área médica e o segundo, para se referir às demais áreas da saúde.

Mais especificamente na área da Audiologia, a Teleprática pode ser utilizada na realização dos seguintes procedimentos: tratamento, triagem e avaliação; aconselhamento, orientação,

Trabalho realizado no Centro de Pesquisas Audiológicas do Hospital de Reabilitação das Anomalias Craniofacias (HRAC) da Universidade de São Paulo - USP - Bauru (SP), Brasil.

(1) Fonoaudióloga do Programa de Triagem Auditiva Neonatal da Universidade Federal de Uberlândia - UFU - Uberlândia (MG), Brasil.

(2) Professora Titular da Faculdade de Odontologia de Bauru da Universidade de São Paulo - USP - Bauru (SP), Brasil.

(3) Doutora, Bolsista de pós-doutorado da Fundação de Apoio à Pesquisa do Estado de São Paulo (FAPESP) pela Universidade de São Paulo - USP - São Paulo (SP), Brasil.

(4) Professor Titular do Departamento de Fonoaudiologia da Faculdade de Odontologia de Bauru da Universidade de São Paulo - USP - Bauru (SP), Brasil.

Endereço para correspondência: Camila Eugênia Zumpano. R. Alexandre Martins Marques, 290, Vigilato Pereira, Uberlândia (MG), Brasil, CEP: 38408-456. E-mail: cazump@yahoo.com.br

Recebido em: 3/3/2009; Aceito em: 20/5/2009 consulta profissional, verificação de equipamentos (aparelhos de amplificação sonora individual - AASI, sistemas de frequência modulada - sistemas FM e implante coclear - IC), atividades de prevenção, entre outros serviços ${ }^{(1,6)}$.

A American Speech-Language-Hearing Association (ASHA) realizou em 2002 uma pesquisa sobre o uso da Teleprática entre os membros associados (audiologistas e terapeutas da fala e linguagem). Participaram deste levantamento 1667 membros (50,5\% audiologistas e $49,5 \%$ terapeutas da fala e linguagem), sendo que apenas $11 \%$ eram usuários deste modelo de atendimento.

Geralmente, o cliente beneficiário da Teleprática mora em áreas suburbanas ou rurais, onde há escassez de profissionais especializados e dificuldade de acesso ao tratamento em grandes centros de referência, devido à distância, custos com transporte e custos indiretos associados à perda da produtividade do trabalho com o tempo de viagem e acompanhamento de familiares.

De acordo com este levantamento realizado pela ASHA (2002), os principais serviços oferecidos pela Teleprática foram: aconselhamento (76\%), acompanhamento e orientação $(71 \%)$, verificação de equipamentos (34\%), atividade preventiva (27\%), tratamento (23\%), triagem (18\%), serviço de tradução online (13\%) e avaliação (11\%).

Alguns pesquisadores utilizaram a tecnologia das telecomunicações para realizar o diagnóstico audiológico remotamente, por meio dos exames de Audiometria, Logoaudiometria, Emissões Otoacústicas (EOA) e Potenciais Evocados 
Auditivos de Tronco Encefálico (PEATE), demonstrando a aplicação e a importância da Teleprática na área da Audio$\operatorname{logia}{ }^{(7-12)}$.

Além do diagnóstico audiológico, a Teleprática pode ser utilizada para a programação remota de dispositivos eletrônicos como, IC e AASI em indivíduos residentes em áreas distantes, onde há falta de profissional especializado e centros de referência ${ }^{(13-15)}$.

No Brasil, até o momento, existem dez centros que realizam a cirurgia e acompanhamento pós-cirúrgico do IC pelo Sistema Único de Saúde (SUS). Estes centros de referência estão concentrados nas regiões Sudeste (cinco), Sul (um), Nordeste (três) e Centro-Oeste (um).

Um destes centros, localizado no interior de São Paulo, é o Centro de Pesquisas Audiológicas (CPA) do Hospital de Reabilitação de Anomalias Craniofacias (HRAC) da Universidade de São Paulo (USP), campus Bauru, o qual possui no momento 600 pacientes implantados, provenientes das diversas regiões do país e que fazem acompanhamento periódico na cidade de Bauru.

A concentração dos centros de IC em algumas regiões leva a necessidade dos pacientes e acompanhantes se deslocarem de suas cidades de origem, o que envolve custos relacionados ao transporte e acomodação, além de interferir nas atividades escolares e/ou profissionais dos pacientes e de seus acompanhantes. Portanto, a programação remota dos sistemas de IC representa um recurso promissor para descentralizar os serviços de saúde, proporcionar o treinamento de profissionais que atuam na área de Audiologia e reduzir custos para o SUS.

Diante do exposto, o objetivo deste estudo foi investigar os recursos necessários para a viabilização da programação remota dos Sistemas de Implante Coclear e verificar os benefícios e limitações para a implementação deste modelo de atendimento nos serviços de saúde auditiva do Brasil.

\section{APRESENTAÇÃO DOS CASOS CLÍNICOS}

Este estudo foi aprovado pelo Comitê de Ética em Pesquisa em Seres Humanos do HRAC/USP, sob ofício n¹94/2006SVAPEPE-CEP. Todos os participantes e responsáveis assinaram o Termo de Consentimento Livre e Esclarecido, após terem recebido informações sobre os objetivos, justificativa e metodologia da pesquisa proposta.

Participaram deste estudo dois sujeitos usuários de IC multicanal, residentes em uma mesma cidade (Brasília ou cidade satélite) e regularmente matriculados e acompanhados no CPA/ HRAC-USP e dois profissionais graduados em Fonoaudiologia que atuam na área de Audiologia.

As características dos sujeitos implantados estão expostas na Tabela 1.
Os participantes graduados em Fonoaudiologia eram: um profissional na área remota (AR), residente em Brasília-DF, com atuação em (re) habilitação auditiva e sem experiência prévia na programação dos sistemas de IC, e um profissional no centro de referência (CR), residente em Bauru-SP, com experiência em programação dos sistemas de IC e com atuação nesta área.

O procedimento foi realizado entre os seguintes centros: CPA/HRAC-USP da cidade de Bauru/SP (centro de referência) e Centro Educacional da Audição e Linguagem Ludovico Pavoni (CEAL-LP), da cidade de Brasília/DF (área remota). A cidade remota selecionada foi Brasília, por possuir um número considerável de sujeitos implantados acompanhados pelo CPA/ HRAC-USP e profissionais que atuam na área de Audiologia.

Os equipamentos utilizados no $\mathrm{CR}$ e na AR foram: telefone, dois computadores com conexão de Internet banda larga, software Custom Sound da Cochlear Corporation®, interfaces e cabos para programação do IC. As especificações do computador pessoal (PC) Intel/Pentium do CR foram processador de 3,00 GHz, 512 MB de memória RAM e sistema operacional Microsoft@ Windows XP (versão 2002), enquanto que o notebook Dell@ Latitude utilizado na AR tinha processador de $980 \mathrm{MHz}, 0,99 \mathrm{~GB}$ de memória RAM e sistema operacional Microsoft@ Windows XP (versão 2002). Inicialmente, foi realizado o treinamento do uso do WebEx da Cochlear Corporation $\AA$, recurso selecionado para possibilitar o controle da área de trabalho desejada na AR. Todos os treinamentos foram realizados sem a presença dos usuários de IC. Durante os treinamentos a comunicação entre os profissionais foi realizada por telefone e chat, via conexão simultânea da Internet.

Este procedimento foi realizado com a finalidade de estruturar os recursos para a execução do procedimento à distância, treinar os fonoaudiólogos no uso dos equipamentos utilizados na programação remota do IC, treinar o fonoaudiólogo da AR para o uso do software Custom Sound e elaborar um roteiro das orientações a serem dadas ao profissional da AR.

O profissional sem experiência na programação dos sistemas de IC recebeu orientação prévia sobre definição, componentes e funcionamento do IC, procedimentos e parâmetros da programação deste dispositivo e etapas da programação.

Ambos os profissionais envolvidos no procedimento, estavam cientes da confidencialidade dos sujeitos implantados, da proficiência no uso dos recursos tecnológicos selecionados para a atividade e do foco na qualidade do atendimento.

Após os cinco treinamentos realizados entre o $\mathrm{CR}$ e a AR e orientações prévias, foi realizada a programação remota do sistema de Implante Coclear nos dois sujeitos selecionados. $\mathrm{O}$ procedimento foi realizado em dois dias consecutivos, sendo um sujeito atendido em cada dia, na AR.

Nesta etapa, o fonoaudiólogo (Participante 1), localizado

Tabela 1. Características demográficas dos sujeitos usuários de implante coclear

\begin{tabular}{|c|c|c|c|c|c|c|c|}
\hline Sujeito & $\begin{array}{c}\text { Etiologia } \\
\text { da DA }\end{array}$ & $\begin{array}{l}\text { Idade na } \\
\text { cirurgia }\end{array}$ & $\begin{array}{l}\text { Inserção dos } \\
\text { eletrodos }\end{array}$ & $\begin{array}{c}\text { Tipo de IC/ } \\
\text { Processador }\end{array}$ & $\begin{array}{l}\text { Tempo de } \\
\text { uso do IC }\end{array}$ & $\begin{array}{l}\text { Idade na programação } \\
\text { remota }\end{array}$ & $\begin{array}{c}\text { Grau de } \\
\text { escolaridade }\end{array}$ \\
\hline 1 & Citomegalovírus & $4 a 8 m$ & total & $24 \mathrm{~K} /$ Sprint* & 10a $9 m$ & 14 anos & Cursando $8^{\circ}$ ano \\
\hline 2 & Meningite & 3a $3 m$ & total & $24 \mathrm{~K} /$ Sprint* & $9 a 4 m$ & 12 anos & Cursando $5^{\circ}$ ano \\
\hline
\end{tabular}

Legenda: $\mathrm{DA}=$ deficiência auditiva; $\mathrm{a}=$ anos; $\mathrm{m}=$ meses; $\mathrm{IC}=$ implante coclear. ${ }^{*}$ Cochlear Corporation 
no CR, conduziu a programação do sistema de IC a distância, orientando e indicando os passos do procedimento para o outro profissional (Participante 2), localizado na AR, quando solicitado e necessário. Os participantes seguiram o roteiro previamente elaborado e enviado ao profissional da AR via e-mail.

Após o término da programação remota, o Participante 2 respondeu um questionário (Anexo 1) previamente elaborado, o qual abordou as vantagens, dificuldades e outros aspectos do procedimento realizado. Os dois sujeitos implantados também responderam um questionário (Anexo 2) comparando o atendimento presencial, realizado nos retornos periódicos ao CPA, ao atendimento remoto. Os questionários foram respondidos pelos sujeitos com o auxílio dos responsáveis e foi aplicado no formato caneta e papel.

O questionário direcionado ao fonoaudiólogo da área remota continha 17 questões, sendo oito no formato de escala graduada de cinco pontos, cinco no formato de múltipla escolha, com alternativas de respostas e quatro discursivas. Já o questionário destinado aos usuários de IC foi dividido em duas partes, a primeira referente ao atendimento presencial e a segunda destinada ao atendimento remoto. Ambas as partes compostas por 12 questões, sendo cinco no formato de escala graduada de cinco pontos e sete no formato de múltipla escolha, com alternativas de respostas. As duas partes foram compostas por 11 questões semelhantes e apenas uma diferente. A questão diferente destinada ao atendimento presencial referiu-se à necessidade de auxílio financeiro para a viagem e acomodação e do atendimento remoto ao grau de diferença entre $o$ atendimento presencial e remoto.

\section{DISCUSSÃO}

Durante o treino do uso do WebEx houve falhas na conexão via Internet e dificuldade no controle da área de trabalho desejada tanto para o participante do CR como da AR. A ausência de outros recursos na AR, como uma webcam e um microfone, ocasionaram a falta de monitoramento pela profissional do CR em relação ao procedimento de programação remota.

Durante a realização das duas sessões de programação remota do IC, o uso do WebEx Support Center possibilitou o início e término das sessões sem interrupção, a troca de controle da área de trabalho desejada, a visualização e o acesso ao computador da área remota, a comunicação entre os participantes por meio do chat e a gravação dos procedimentos através do recurso disponibilizado pelo WebEx. A manipulação do WebEx pelos Participantes 1 e 2 durante os treinamentos foi fundamental para a posterior realização da programação remota. No decorrer das sessões, não existiram falhas na conexão da Internet de banda larga.

Quanto ao uso do software Custom Sound utilizado na programação do IC da Cochlear Corporation ${ }^{\circledR}$, o Participante 2 apresentou dificuldades em alguns dos procedimentos que haviam sido detalhados nas orientações prévias enviadas ao mesmo. As maiores dificuldades relacionaram-se a procedimentos específicos da programação do IC, de forma que o Participante 2 solicitava auxílio via chat passo a passo. Isso ocasionou no aumento do tempo da sessão e foi necessária a intervenção do Participante 1 para finalizar a mesma, já que o primeiro sujeito implantado apresentou cansaço.

Já na segunda sessão, o Participante 2 realizou todos os procedimentos descritos nas orientações prévias. No entanto, evidenciou algumas dúvidas semelhantes àquelas notadas na primeira sessão, o que levou ao atraso do procedimento e cansaço do segundo sujeito. Porém, a sessão foi concluída pelo Participante 2.

A duração aproximada da primeira sessão foi de uma hora e 40 minutos e da segunda, uma hora e 15 minutos. Normalmente, a duração média no CR é de 40 minutos, de acordo com a faixa etária dos sujeitos estudados.

Após cada sessão, o Participante 1 entrou em contato com os responsáveis pelos sujeitos implantados, por meio de telefone, para explicar o uso dos novos programas.

Quanto ao questionário direcionado ao profissional da área remota, verificou-se que o Participante 2 apresentava um domínio básico da tecnologia da telecomunicação, utilizando em sua rotina, apenas o $e$-mail e a Internet. As dificuldades pontuadas pelo mesmo durante o treinamento do WebEx, graduadas como moderadas, foram a troca de controle da área de trabalho desejada e a compreensão das orientações dadas pelo Participante 1.

Em relação às orientações prévias, o Participante 2 relatou que lhe ofereceram ajuda moderada durante a programação remota e que poucas informações não foram descritas como, por exemplo, posicionamento dos sujeitos e instalação dos equipamentos.

As dificuldades durante a programação remota dos dois sujeitos foram relativas aos procedimentos específicos do mapeamento do IC. O Participante 2 relatou que durante as sessões atuava como "técnico", não compreendendo os passos. Por esta razão, é imprescindível que o profissional do CR tenha experiência na programação de sistema de IC, com o intuito de auxiliar e monitorar o profissional da AR, no caso de possíveis dúvidas e questionamentos.

De modo geral, o Participante 2 avaliou a programação remota de modo positivo, relatando contribuição em sua atuação profissional como reabilitador, economia de gastos para os sujeitos e familiares e a possibilidade do acompanhamento dos usuários de IC, sem a necessidade de se ausentar da escola e terapia.

Após a realização dos dois procedimentos, o Participante 2 classificou como "bastante" seu grau de dificuldade para realizar a programação remota do IC sem o auxílio do Participante 1 , e grande possibilidade em participar de outro procedimento envolvendo os recursos da teleprática.

Quanto ao questionário direcionado aos sujeitos implantados (Quadro 1), verificou-se que a economia de tempo e gastos relacionados ao deslocamento, acomodação e necessidade de não se ausentar da escola ou trabalho, foram os principais benefícios da programação remota.

Ao comparar o atendimento presencial ao remoto, os sujeitos implantados informaram que o atendimento à distância foi mais demorado e que apresentou pouca diferença em relação ao tradicional.

Em ambos, os usuários de IC não apresentaram dificuldade na compreensão das orientações dadas pelos profissionais 
Quadro 1. Resultados do questionário direcionado aos usuários de implante coclear

\begin{tabular}{|c|c|c|c|c|}
\hline \multirow{2}{*}{ Aspectos avaliados } & \multicolumn{2}{|c|}{ Atendimento presencial } & \multicolumn{2}{|c|}{ Atendimento remoto } \\
\hline & Sujeito 1 & Sujeito 2 & Sujeito 1 & Sujeito 2 \\
\hline $\begin{array}{l}\text { 1. Tempo para chegar ao local de } \\
\text { atendimento }\end{array}$ & 5 a $10 h$ & Mais de $10 \mathrm{~h}$ & Menos de $1 \mathrm{~h}$ & Menos de $1 \mathrm{~h}$ \\
\hline $\begin{array}{l}\text { 2. Meio de transporte para } \\
\text { chegar ao local de atendimento }\end{array}$ & Avião & Carro & Carro & Carro \\
\hline $\begin{array}{l}\text { 3. Necessidade de auxílio } \\
\text { financeiro }\end{array}$ & $\begin{array}{l}\text { Razoável auxílio foi } \\
\text { necessário }\end{array}$ & $\begin{array}{l}\text { Nenhum auxílio foi } \\
\text { necessário }\end{array}$ & Não se aplica & Não se aplica \\
\hline $\begin{array}{l}\text { 4. Necessidade de } \\
\text { acompanhante }\end{array}$ & Sim (mãe) & Sim (pai) & Sim (mãe) & Sim (mãe) \\
\hline $\begin{array}{l}\text { 5. Necessidade de se ausentar } \\
\text { da escola }\end{array}$ & Sim (3 dias) & Sim (3 dias) & Não & Não \\
\hline $\begin{array}{l}\text { 6. Tempo na sala de espera até } \\
\text { ser atendido }\end{array}$ & $30 \min$ a $1 \mathrm{~h}$ & Menos de $30 \mathrm{~min}$ & Menos de $30 \mathrm{~min}$ & Menos de $30 \mathrm{~min}$ \\
\hline 7. Tempo para programar o IC & Menos de $30 \mathrm{~min}$ & $30 \min$ a $1 \mathrm{~h}$ & $1 \mathrm{a} 2 \mathrm{~h}$ & $1 \mathrm{a} 2 \mathrm{~h}$ \\
\hline $\begin{array}{l}\text { 8. } N^{\circ} \text { de sessões para programar } \\
\text { o IC }\end{array}$ & 1 sessão & 1 sessão & 1 sessão & 1 sessão \\
\hline $\begin{array}{l}\text { 9. Grau de dificuldade das } \\
\text { orientações do profissional }\end{array}$ & Nenhuma & Nenhuma & Nenhuma & Nenhuma \\
\hline $\begin{array}{l}\text { 10. Grau de satisfação com os } \\
\text { novos programas }\end{array}$ & Bastante & Bastante & Bastante & Bastante \\
\hline $\begin{array}{l}\text { 11. Grau de satisfação com o } \\
\text { atendimento }\end{array}$ & Muita & Muita & Muita & Bastante \\
\hline $\begin{array}{l}\text { 12. Probabilidade de indicação } \\
\text { do serviço a outras pessoas }\end{array}$ & Muita & Muita & Bastante & Muita \\
\hline $\begin{array}{l}\text { 13. Grau de diferença entre } \\
\text { atendimento presencial e remoto }\end{array}$ & Não se aplica & Não se aplica & Pouca & Pouca \\
\hline
\end{tabular}

Legenda: $\mathrm{h}=$ hora; $\min =$ minuto

(Participantes 1 e 2); revelaram satisfação com o atendimento realizado e grande probabilidade de indicar o serviço a outras pessoas.

É importante comentar que as etapas que antecederam às sessões de programação remota, referentes ao treino do uso do WebEx, foram fundamentais para o conhecimento e prática desta tecnologia, já que neste período, os Participantes 1 e 2 apresentaram dificuldade na manipulação de alguns dos recursos disponíveis. Entretanto, tal dificuldade foi sanada com a prática. Como referido pela $\mathrm{ASHA}^{(1-2)}$, o conhecimento e a proficiência no uso dos diversos tipos de tecnologia das telecomunicações são requisitos necessários para a realização de serviços via Teleprática.

A programação remota dos sistemas de IC consiste em uma aplicação clínica inovadora da Teleprática na área de Audiologia, visto a escassez de literatura sobre este modelo de atendimento. Alguns pesquisadores realizaram a programação remota do IC em dez pacientes do Children's Hospital of Philadelphia (CHOP). Os profissionais da área remota foram responsáveis pela videoconferência e programação do equipamento. Os pacientes e familiares aprovaram o serviço à distância e os professores demonstraram interesse em expandir a disponibilidade do procedimento ${ }^{(13)}$.

No presente estudo, a programação remota do IC proporcionou o treinamento do profissional da área da (re) habilitação auditiva, o acesso ao serviço especializado aos sujeitos implantados sem a necessidade de deslocar-se e ausentar-se de suas atividades escolares; bem como economia para o SUS em relação à receita destinada ao Tratamento Fora do Domicílio (TFD), de acordo com a portaria da Secretaria de Atenção à Saúde do Ministério da Saúde, n 55 de 24/02/1999.

Entretanto, algumas dificuldades e limitações devem ser superadas como, por exemplo, falhas na conexão, competência técnica no uso da tecnologia, falta de recursos tecnológicos nas áreas remotas, falta de contato visual entre a profissional do centro de referência e os sujeitos usuários de IC e conhecimento básico anterior sobre programação de IC.

Segundo a ASHA, a satisfação do cliente e dos profissionais envolvidos no modelo de atendimento à distância é um aspecto importante que deve ser considerado ${ }^{(2)}$. Neste estudo, ao analisar as respostas do questionário direcionado aos usuários de IC, observou-se que os dois sujeitos revelaram um alto grau de satisfação com os novos programas e com o atendimento realizado na área remota. Quando os sujeitos compararam o atendimento presencial ao remoto, relataram maior tempo necessário para a realização da programação do IC à distância. Este fato pode ser explicado pela falta de troca de controle da área de trabalho com maior frequência. Outro fator a ser considerado é a necessidade de mais treinamentos do software de programação do IC, o que não foi realizado devido ao uso do equipamento na rotina do CPA. Uma alternativa seria a visita e treinamento do profissional da AR no CR. Além 
disso, a programação remota do IC poderia ser realizada pelo profissional do CR com a participação do profissional da AR, junto ao paciente, apenas para a instalação dos equipamentos necessários, conexão do processador de fala e orientação ao paciente sobre a forma de responder ao mapeamento.

Em alguns estudos, o profissional da área remota atuou apenas como um intermediário do procedimento, sendo este realizado pelo profissional do centro de referência ${ }^{(9,11)}$.

A programação remota dos sistemas de IC, em um maior número de indivíduos, mais treinamentos do profissional da área remota sobre uso do software de programação do IC e a aquisição de recursos tecnológicos pelos serviços de saúde (webcam, fone, outros), são alguns dos fatores que devem ser considerados antes da aplicação do procedimento em ampla escala.

\section{COMENTÁRIOS FINAIS}

A programação remota dos sistemas de IC representa um recurso promissor e inovador na área da Audiologia, a qual proporcionou possibilidade de descentralização dos serviços de saúde e redução de custos para o SUS no acompanhamento de pacientes implantados.

Este estudo evidenciou as vantagens e dificuldades relacionadas à aplicação da Teleprática nos serviços de saúde auditiva do país.

A programação remota do IC demonstrou ser um modelo de complementação ao acompanhamento dos sujeitos usuários deste dispositivo efetivo, viável e inovador no Brasil. Entretanto, ainda é necessário realizar mais pesquisas para viabilizar o uso em escala nacional.

\begin{abstract}
The aims of the present study were to investigate the required resources to allow the remote programming of Cochlear Implants (CI), and to assess the benefits and limitations of implementing this model of programming in Brazilian hearing health care services. The study was conducted with two multichannel CI recipients from the Audiological Research Center (CPA/HRAC) of the University of São Paulo - Brazil (USP) and two audiologists. One of the professionals was based at the CPA/HRAC-USP, which was the reference center (RC), and the other audiologist was based at an Audiology Center located more than 900mi away from the RC, which was called remote area (RA). The audiologist from RA had no previous experience in programming CI devices. The procedure consisted in remotely training the RA audiologist. After the conference call, the RA professional and the implanted subjects answered a previously elaborated questionnaire. The study showed the following advantages of applying Cochlear Implant Remote Programming: decentralization of the hearing health care services, training and enabling of professionals, cost reduction for the Public Health Care System (SUS), and systematic monitoring of implanted patients. Conference call is an effective, feasible and innovative model of monitoring cochlear implant's recipients in Brazil. However more studies are necessary in order to make the remote programming available nationwide.
\end{abstract}

Keywords: Remote consultation/methods; Cochlear implant; Hearing loss; Telemedicine/instrumentation; Case reports

\section{REFERÊNCIAS}

1. American Speech-Language-Hearing Association. Audiologists providing clinical services via telepractice: position statement [Internet]. [cited 2007 Dec 6]. Available from: http://www.asha.org/docs/pdf/ PS2005-00029.pdf

2. American Speech-Language-Hearing Association. Knowledge and skills needed by audiologists providing clinical services via telepractice. ASHA Telepractice Working Group. [Knowledge and Skills] [Internet]. [cited 2007 Dec 6]. Available from: http://www.asha.org/docs/pdf/ KS2005-00084.pdf

3. Krumm M. Audiology telepractice moves from theory to treatment. The ASHA Leader 2005;(45):22-3.

4. AHRQ - Agency for Healthcare Research and Quality. Telemedicine for the medicare population. Summary, Evidence Report/Technology Assessment: Number 24 [Internet]. Rockville, MD; 2001 [cited 2007 Dec 6]. Available from: http://www.ahrq.gov/clinic/epcsums/ telemedsum.htm

5. ATA defining telemedicine [internet]. [cited 2007 Aug 28]. Available from: http://www.atmeda.org/news/definition.html

6. American Speech-Language-Hearing Association. Survey of telepractice use among audiologists and speech-language pathologists. Rockville, MD: American Speech-Language-Hearing Association; 2002.
7. Givens GD, Elangovan S. Internet application to tele-audiology "nothin' but net". Am J Audiol. 2003;12(2):59-65.

8. Givens GD, Blanarovich A, Murphy T, Simmons S, Blach D, Elangovan S. Internet-based tele-audiometry system for the assessment of hearing: a pilot study. Telemed J E Health. 2003;9(4):375-8.

9. Elangovan S. Telehearing and the internet. Semin Hear. 2005;26(1):1925

10. Ribera JE. Interjudge reliability and validation of telehealth applications of the hearing in noise test. Semin Hear. 2005;26(1):13-8.

11. Krumm M. Audiology telemedicine. J Telemed Telecare. 2007;13(5):224-9.

12. Krumm M, Ribera J, Klich R. Providing basic hearing tests using remote computing technology. J Telemed Telecare. 2007;13(8):406-10.

13. Franck K, Pengelly M, Zerfoss S. Telemedicine offers remote cochlear implant programming. Volta Voices. 2006;13(1):16-9.

14. Wesendahl T. Hearing aid fitting: application of telemedicine in audiology. Int Tinnitus J. 2003;9(1):56-8.

15. Ferrari DV. Remote programming and verification as a mean to improve quality of hearing aid fitting. In: Rasmussen AN, Paulsen T, Andersen T, Larsen CB, editors. Hearing aid fitting. Centertryk: Danavox Jubilee Foundation; 2006; p. 531-44. 
Anexo 1. Questionário - Programação remota (direcionado ao profissional)

Favor responder as questões abaixo, completando os espaços e assinalando a resposta mais adequada. Essas informações são importantes para nossa pesquisa.

1) Quantos pacientes atendidos por você (terapia fonoaudiológica) são acompanhados pelo CPA/Bauru?

2) Quantos pacientes do CEAL/Brasília são acompanhados pelo CPA/Bauru?

3) Quantos pacientes do CEAL/Brasília são acompanhados por outros Centros?

4) Quais tecnologias das telecomunicações você já tem domínio?

$$
\begin{aligned}
& \square \text { e-mail } \\
& \square \text { chat } \\
& \square \text { comunicação por áudio (MSN/Skype) } \\
& \square \text { comunicação por webcam } \\
& \square \text { outros: }
\end{aligned}
$$

5) Você já tinha experiência em teleassistência/teleeducação?
$\square$ nenhuma
$\square$ pouca
$\square$ moderada
$\square$ bastante
$\square$ muita

6) Você já tinha experiência em programação dos sistemas de Implante Coclear?
$\square$ nenhuma
$\square$ pouca
$\square$ moderada
$\square$ bastante
$\square$ muita

7) Quais foram suas dificuldades durante o treinamento do WebEx?

$\square$ uso do chat

$\square$ falhas na conexão

$\square$ início da sessão

$\square$ término da sessão

$\square$ controle da área de trabalho desejada

$\square$ compreensão das orientações da especialista de Bauru

$\square$ outras:

8) Qual o seu grau dificuldade no uso do WebEx da Cochlear?

$\square$ nenhuma

$\square$ pouca

$\square$ moderada

$\square$ bastante

$\square$ muita

9) A apresentação em Power Point, com algumas orientações prévias sobre programação de Implante Coclear, Ihe auxiliou durante a teleassistência?

$\square$ nenhuma ajuda

$\square$ pouca ajuda

$\square$ moderada ajuda

$\square$ bastante ajuda

$\square$ muita ajuda

10) Quais foram suas dúvidas sobre as orientações prévias, em Power Point?

$\square$ compontes do Implante Coclear

$\square$ funcionamento do Implante Coclear

$\square$ procedimentos do mapeamento

$\square$ parâmetros do mapeamento

$\square$ etapas da programação remota do Implante Coclear

$\square$ outras:
11) Na apresentação em Power Point, ficou faltando alguma informação que foi relevante durante a teleassistência?
$\square$ nenhuma informação faltou
$\square$ pouca informação faltou
$\square$ moderada informação faltou
$\square$ bastante informação faltou
$\square$ muita informação faltou

12) Quais foram suas dificuldades durante a programação remota do paciente 1 ?

$\square$ uso do chat

$\square$ falhas na conexão

$\square$ conectar o processador

$\square$ troca de controle da área de trabalho desejada

$\square$ compreensão das orientações da especialista de Bauru

$\square$ observar as respostas do paciente

$\square$ realizar procedimento específico do mapeamento (telemetria, pesquisa $\mathrm{T}$ e C)

$\square$ outras:

13) Quais foram suas dificuldades durante a programação remota do paciente 2 ?

$\square$ uso do chat

$\square$ falhas na conexão

$\square$ conectar o processador

$\square$ troca de controle da área de trabalho desejada

$\square$ compreensão das orientações da especialista de Bauru

$\square$ observar as respostas do paciente

$\square$ realizar procedimento específico do mapeamento (telemetria, pesquisa $\mathrm{T}$ e C)

$\square$ outras:

14) Após os dois procedimentos, qual o grau de dificuldade para você realizar a programação do Implante Coclear sem o auxílio de uma especialista?
$\square$ nenhuma
$\square$ pouca
$\square$ moderada
$\square$ bastante
$\square$ muita

15) Os conhecimentos adquiridos durante a teleassistência e teleeducação vão te auxiliar na sua atuação profissional?

$\square$ nenhuma ajuda

$\square$ pouca ajuda

$\square$ moderada ajuda

$\square$ bastante ajuda

$\square$ muita ajuda

16) Na sua opinião quais as vantagens de se utilizar a teleassistência?

17) Qual a possibilidade de você participar de outro procedimento de teleassistência?

$\square$ nenhuma

$\square$ pouca

$\square$ moderada

$\square$ bastante

$\square$ muita 
Anexo 2. Questionário - Programação remota (direcionado ao paciente)

Favor responder as questões abaixo, completando os espaços e assinalando a resposta mais adequada. Esse questionário fala sobre o atendimento que você teve no CPA/Bauru. Essas informações são importantes para nossa pesquisa. Colabore.

Nome:

Idade: Sexo: ( ) Masculino ( ) Feminino

Escolaridade:

Faz terapia fonoaudiológica? ( ) Sim （ ) Não Quantas vezes por semana?

Paciente: ( ) 1

Atendimento presencial (Bauru):

\begin{tabular}{|c|c|}
\hline 1. Quanto tempo você levou para chegar a Bauru? & $\begin{array}{l}\square \text { menos de } 1 \text { hora } \\
\square 1 \text { a } 5 \text { horas } \\
\square 5 \text { a } 10 \text { horas } \\
\square \text { mais de } 10 \text { horas }\end{array}$ \\
\hline 2. Qual meio de transporte você usou para chegar até Bauru? & $\begin{array}{l}\square \text { carro } \\
\square \text { ônibus } \\
\square \text { avião } \\
\square \text { táxi } \\
\square \text { outros: }\end{array}$ \\
\hline $\begin{array}{l}\text { 3. Você necessitou de auxílio financeiro para a viagem e acomodações (auxílio } \\
\text { do Tratamento Fora Domicílio do SUS)? }\end{array}$ & $\begin{array}{l}\square \text { nenhum auxílio foi necessário } \\
\square \text { pouco auxílio foi necessário } \\
\square \text { razoável auxílio foi necessário } \\
\square \text { bastante auxílio foi necessário } \\
\square \text { muito auxílio foi necessário }\end{array}$ \\
\hline 4. Você veio ao atendimento sozinho? & $\begin{array}{l}\square \text { sim } \\
\square \text { não, acompanhado da mãe } \\
\square \text { não, acompanhado do pai } \\
\square \text { não, acompanhado dos pais } \\
\square \text { não, acompanhado por outros parentes }\end{array}$ \\
\hline 5. Você teve que se ausentar da escola para vir ao atendimento? & $\begin{array}{l}\square \text { sim, quantos dias: } \\
\square \text { não }\end{array}$ \\
\hline 6. Quanto tempo você ficou na sala de espera até ser atendido? & $\begin{array}{l}\square \text { menos de } 30 \text { minutos } \\
\square 30 \text { minutos a } 1 \text { hora } \\
\square 1 \text { a } 2 \text { horas } \\
\square \text { mais de } 2 \text { horas }\end{array}$ \\
\hline 7. Quanto tempo levou para programar seu Implante? & $\begin{array}{l}\square \text { menos de } 30 \text { minutos } \\
\square 30 \text { minutos a } 1 \text { hora } \\
\square 1 \text { a } 2 \text { horas } \\
\square \text { mais de } 2 \text { horas }\end{array}$ \\
\hline 8. Quantas sessões foram necessárias para programar o seu Implante? & $\begin{array}{l}\square 1 \text { sessão } \\
\square 2 \text { sessões } \\
\square 3 \text { sessões } \\
\square \text { mais de } 3 \text { sessões }\end{array}$ \\
\hline 9. Qual o grau de dificuldade das orientações dadas pela fonoaudióloga? & $\begin{array}{l}\square \text { nenhuma } \\
\square \text { pouca } \\
\square \text { moderada } \\
\square \text { bastante } \\
\square \text { muita }\end{array}$ \\
\hline 10. Qual o grau de satisfação com os novos programas do seu Implante? & $\begin{array}{l}\square \text { nenhuma } \\
\square \text { pouca } \\
\square \text { moderada } \\
\square \text { bastante } \\
\square \text { muita }\end{array}$ \\
\hline 11. Qual o grau de satisfação com o atendimento realizado pela fonoaudióloga? & $\begin{array}{l}\square \text { nenhuma } \\
\square \text { pouca } \\
\square \text { moderada } \\
\square \text { bastante } \\
\square \text { muita }\end{array}$ \\
\hline 12. Qual a probabilidade de você indicar este serviço à outra pessoa? & $\begin{array}{l}\square \text { nenhuma } \\
\square \text { pouca } \\
\square \text { moderada } \\
\square \text { bastante } \\
\square \text { muita }\end{array}$ \\
\hline
\end{tabular}


Esse questionário fala sobre o atendimento que você teve no CEAL/Brasília Atendimento remoto (Brasília):

\begin{tabular}{|c|c|}
\hline 1. Quanto tempo você levou para chegar até ao CEAL? & $\begin{array}{l}\square \text { menos de } 1 \text { hora } \\
\square 1 \text { a } 5 \text { horas } \\
\square 5 \text { a } 10 \text { horas } \\
\square \text { mais de } 10 \text { horas }\end{array}$ \\
\hline 2. Qual meio de transporte você usou para chegar até o CEAL? & $\begin{array}{l}\square \text { carro } \\
\square \text { ônibus } \\
\square \text { avião } \\
\square \text { táxi } \\
\square \text { outros: } \\
\end{array}$ \\
\hline 3. Você veio ao atendimento sozinho? & $\begin{array}{l}\square \text { sim } \\
\square \text { não, acompanhado da mãe } \\
\square \text { não, acompanhado do pai } \\
\square \text { não, acompanhado dos pais } \\
\square \text { não, acompanhado por outros parentes }\end{array}$ \\
\hline 4. Você teve que se ausentar da escola para vir ao atendimento? & $\begin{array}{l}\square \text { sim, quantos dias: } \\
\square \text { não }\end{array}$ \\
\hline 5. Quanto tempo você ficou na sala de espera até ser atendido? & $\begin{array}{l}\square \text { menos de } 30 \text { minutos } \\
\square 30 \text { minutos a } 1 \text { hora } \\
\square 1 \text { a } 2 \text { horas } \\
\square \text { mais de } 2 \text { horas }\end{array}$ \\
\hline 6. Quanto tempo levou para programar seu Implante? & $\begin{array}{l}\square \text { menos de } 30 \text { minutos } \\
\square 30 \text { minutos a } 1 \text { hora } \\
\square 1 \text { a } 2 \text { horas } \\
\square \text { mais de } 2 \text { horas }\end{array}$ \\
\hline 7. Quantas sessões foram necessárias para programar o seu Implante? & $\begin{array}{l}\square 1 \text { sessão } \\
\square 2 \text { sessões } \\
\square 3 \text { sessões } \\
\square \text { mais de } 3 \text { sessões }\end{array}$ \\
\hline 8. Qual o grau de dificuldade das orientações dadas pela fonoaudióloga? & $\begin{array}{l}\square \text { nenhuma } \\
\square \text { pouca } \\
\square \text { moderada } \\
\square \text { bastante } \\
\square \text { muita }\end{array}$ \\
\hline 9. Qual o grau de satisfação com os novos programas do seu Implante? & $\begin{array}{l}\square \text { nenhuma } \\
\square \text { pouca } \\
\square \text { moderada } \\
\square \text { bastante } \\
\square \text { muita }\end{array}$ \\
\hline 10. Qual o grau de satisfação com o atendimento realizado pela fonoaudióloga? & $\begin{array}{l}\square \text { nenhuma } \\
\square \text { pouca } \\
\square \text { moderada } \\
\square \text { bastante } \\
\square \text { muita }\end{array}$ \\
\hline $\begin{array}{l}\text { 11. Qual a probabilidade de você indicar esta forma de atendimento à distância } \\
\text { a outra pessoa? }\end{array}$ & $\begin{array}{l}\square \text { nenhuma } \\
\square \text { pouca } \\
\square \text { moderada } \\
\square \text { bastante } \\
\square \text { muita }\end{array}$ \\
\hline 12. Qual o grau de diferença entre o atendimento realizado em Bauru e Brasília? & $\begin{array}{l}\square \text { nenhuma } \\
\square \text { pouca } \\
\square \text { moderada } \\
\square \text { bastante } \\
\square \text { muita }\end{array}$ \\
\hline
\end{tabular}

